\title{
Hadronic Matter with Internal Symmetries and its Consequences: An Expanding Hadronic Gas *
}

\author{
Ludwik Turko \\ Institute of Theoretical Physics, University of Wrocław, \\ Pl.Maksa Borna 9, 50-204 Wrocław, Poland
}

\begin{abstract}
We consider an ideal gas of massive hadrons in thermal and chemical equilibrium. The gas expands longitudinally in accordance with Bjorken law. Strangeness and baryon number conservation is taken into account. This gas has different features as compared to the pion gas.
\end{abstract}

Let us consider a heavy ion collision which goes through the intermediate state of an hadronic matter being in the thermal and chemical equilibrium. This matter can be considered as a gas which expands longitudinally according to a hydrodynamical evolution. In the simplest case the hadronic matter is supposed to be made of non - interacting massless pions [1]. However an assumption of a thermodynamical equilibrium leads to the conclusions that a system under consideration should consist of all species of hadrons allowed by conditions of the equilibrium. We are going to consider an ideal gas consisting of all species of hadrons up to $\Omega^{-}$baryon. These realistic hadrons need that conservation laws due to an internal symmetries should be taken into account.

The aim of this talk is to present some of pecularities of this more realistic hadronic gas as compared to the simple pion gas. Some of these comparison were published before to study $J / \Psi$ suppression patterns [2].

For an ideal hadron gas in thermal and chemical equilibrium, which consists of $l$ species of particles, energy density $\epsilon$, baryon number density $n_{B}$, strangeness density $n_{S}$ and entropy density $s$ read ( $\hbar=c=1$ always)

$$
\epsilon=\frac{1}{2 \pi^{2}} \sum_{i=1}^{l}\left(2 s_{i}+1\right) \int_{0}^{\infty} \frac{d p p^{2} E_{i}}{\exp \left\{\frac{E_{i}-\mu_{i}}{T}\right\}+g_{i}},
$$

*Invited lecture at the "Advanced NATO Workshop: Hot Hadronic Matter: Theory and Experiment", Divonne-les-Bains, June 27 - July 1, 1994. 


$$
\begin{gathered}
n_{B}=\frac{1}{2 \pi^{2}} \sum_{i=1}^{l}\left(2 s_{i}+1\right) \int_{0}^{\infty} \frac{d p p^{2} B_{i}}{\exp \left\{\frac{E_{i}-\mu_{i}}{T}\right\}+g_{i}} \\
n_{S}=\frac{1}{2 \pi^{2}} \sum_{i=1}^{l}\left(2 s_{i}+1\right) \int_{0}^{\infty} \frac{d p p^{2} S_{i}}{\exp \left\{\frac{E_{i}-\mu_{i}}{T}\right\}+g_{i}}, \\
s=\frac{1}{6 \pi^{2} T^{2}} \sum_{i=1}^{l}\left(2 s_{i}+1\right) \int_{0}^{\infty} \frac{d p p^{4}}{E_{i}} \frac{\left(E_{i}-\mu_{i}\right) \exp \left\{\frac{E_{i}-\mu_{i}}{T}\right\}}{\left(\exp \left\{\frac{E_{i}-\mu_{i}}{T}\right\}+g_{i}\right)^{2}}
\end{gathered}
$$

where $E_{i}=\left(m_{i}^{2}+p^{2}\right)^{1 / 2}$ and $m_{i}, B_{i}, S_{i}, \mu_{i}, s_{i}$ and $g_{i}$ are the mass, baryon number, strangeness, chemical potential, spin and a statistical factor of specie $i$ respectively (we treat an antiparticle as a different specie). And $\mu_{i}=B_{i} \mu_{B}+S_{i} \mu_{S}$, where $\mu_{B}$ and $\mu_{S}$ are overall baryon number and strangeness chemical potentials respectively.

In general, all densities of the left sides of eqs.1a-d are functions of time during the cooling of the hadron gas. This means that gas parameters such as temperature and chemical potentials should be functions of time. We would like to obtain these functions explicite. This can be done by solving numerically the system of equations for entropy density $s$, baryon number density $n_{B}$ and strangeness density $n_{S}$, with $s, n_{B}$ and $n_{S}$ given as time dependent quantities. From the Bjorken model we have the following solution for the longitudinal expansion $[1,5]$

$$
s(t)=\frac{s_{0} t_{0}}{t}, \quad n_{B}(t)=\frac{n_{B}^{0} t_{0}}{t},
$$

where $s_{0}$ and $n_{B}^{0}$ are initial densities of the entropy and the baryon number respectively. The overall strangeness is equal to zero during all the evolution. So to solve $(1 \mathrm{~b}, \mathrm{c}, \mathrm{d})$ with $s$ and $n_{B}$ given by $(2)$ and $n_{S}=0$, we need to know initial values $s_{0}$ and $n_{B}^{0}$. To estimate initial baryon number density $n_{B}^{0}$ we use experimental results of $[3]$.

These results are for S-S collisions, but since there are no data on baryon multiplicities for heavier nuclei we have to evaluate them in some way. We assume that the baryon multiplicity per unit rapidity in the CRR is proportional to the number of participating nucleons. For a sulphur-sulphur collision we have $d N_{B} / d y \cong 6$ and 64 participating nucleons [3]. For an $\mathrm{O}-\mathrm{U}$ collision we can roughly estimate the number of participating nucleons at $16+58=74$. The second factor of the sum has been obtained by the following assumption: since an oxygen nucleon is much smaller than an uranium one, we can approximate the part of the uranium, through which the oxygen passes, by the cylinder of the volume equal to $\pi R_{O}^{2} \cdot 2 R_{U}$. The same procedure can be applied to the $\mathrm{S}-\mathrm{U}$ case. Here we obtain $32+93=125$ participants. Therefore, we have $d N_{B} / d y \cong 7$ and $d N_{B} / d y \cong 11.7$ for O-U and S-U collisions respectively. Having taken the initial volume in the CRR equal to $\pi R_{A}^{2} \cdot 1$ $\mathrm{fm}$, we arrive at $n_{B}^{0} \cong 0.25 \mathrm{fm}^{-3}$ for both cases.

To find $s_{0}$, first we have to solve $(1 \mathrm{a}, \mathrm{b}, \mathrm{c})$ with respect to $T, \mu_{S}$ and $\mu_{B}$, where we put $\epsilon=\epsilon_{0}$ and so on. For $\epsilon_{0}$ we have taken estimates given in [4]. 
As a result, we have obtained $T_{0} \cong 212 \mathrm{MeV}, \mu_{S}^{0} \cong 34.6 \mathrm{MeV}$ and $\mu_{B}^{0} \cong 133$ $\mathrm{MeV}$ for the $\mathrm{O}-\mathrm{U}$ collision $\left(\epsilon_{0}=2.5 \mathrm{GeV} / \mathrm{fm}^{3}\right)$ and $T_{0} \cong 209.1 \mathrm{MeV}, \mu_{S}^{0} \cong 36.7 \mathrm{MeV}$ and $\mu_{B}^{0} \cong 143.3 \mathrm{MeV}$ for the $\mathrm{S}-\mathrm{U}$ collision $\left(\epsilon_{0}=2.3 \mathrm{GeV} / \mathrm{fm}^{3}\right)$. Then, from $(1 \mathrm{~d})$ we have $s_{0} \cong 13.68 \mathrm{fm}^{-3}$ for $\mathrm{O}-\mathrm{U}$ and $s_{0} \cong 12.74 \mathrm{fm}^{-3}$ for S-U. Now, having put (2) and $n_{S}=0$ into $(1 \mathrm{~b}, \mathrm{c}, \mathrm{d})$, we can solve them numerically to obtain $T, \mu_{S}$ and $\mu_{B}$ as functions of time. Our results are presented in fig.1, where solid, long-dashed and dashed lines mean the temperature, the strangeness chemical potential and the baryon chemical potential respectively. Fig. 1 shows results for $S-S$ collision initial conditions. The time scale is chosen in a way which enables the temperature to reach the freez-out at $140 \mathrm{MeV}$. This corresponds to the freez-out time equal to $t_{\text {f.o. }} \cong 10.4 \mathrm{fm}$.

The solution for the temperature function can be approximated by an expression of the form

$$
T(t)=T_{0} \cdot\left(\frac{1}{t}\right)^{a}
$$

where

$T_{0}=212.4 \mathrm{MeV}, a=0.178$ for $O-U$ system

and

$T_{0}=209.7 \mathrm{MeV}, a=0.179$ for $S-S$ system.

We can see that all above expressions have the form known from the solution for the longitudinal expansion of a baryonless gas with the sound velocity constant, namely [5]

$$
T(t)=T_{0} \cdot\left(\frac{1}{t}\right)^{c_{s}^{2}}
$$

where $c_{s}$ is the sound velocity and we put the initial time $t_{0}$ equal to $1 \mathrm{fm}$. We have checked that for $n_{B}=0$ results for the temperature function are very similar to those in eq.3. And the following approximations of the temperature function hold

$$
\begin{aligned}
& T(t)=215.2 \cdot\left(\frac{1}{t}\right)^{0.180} \\
& T(t)=208.9 \cdot\left(\frac{1}{t}\right)^{0.172} .
\end{aligned}
$$

We can see that the power in eqs. 3 and 4 is around 0.18 . Therefore a question arises: is this value connected in any way with the sound velocity of the hadron gas? For the baryonless case the answer is positive. We have checked this computing straightforward $c_{s}^{2}=s /\left(T \frac{\partial s}{\partial T}\right)$, which is the value of the sound velocity squared for a baryonless gas [5]. The results are presented in fig.2. For comparision, the square of the sound velocity of a pion gas is also depicted (dashed lines). We can see that in the range of the temperature $200-140 \mathrm{MeV}$ the square of the speed of sound equals 0.17-0.18 indeed. We have also found a region of the temperature where the sound velocity decreases as the temperature increases. Nevertheless, the condition for the stability of the expansion [6], $d / d T\left(s c_{s} / T\right)>0$ is still valid because $s c_{s} / T$ is an increasing function of time. 


\section{Acknowledgments}

It is pleasure to thank Prof. J.Rafelski for his kind invitation to contribute to this workshop and for his warm hospitality.

\section{References}

[1] J.D.Bjorken, Phys.Rev.D 27 (1983) 140

[2] D.Prorok and L.Turko, Z.Phys.C - Particles and Fields 61 (1994) 109

[3] H.Ströbele (NA35 Collab.), Nucl.Phys.A 525 (1991) 59c

[4] M.C.Abreu et al. (NA38 Collab.), Nucl.Phys.A 544 (1992) 209c

[5] J.Cleymans, R.V.Gavai and E.Suhonen, Phys.Rep.130 (1986) 217

[6] G.Baym et al., Nucl.Phys.A 407 (1983) 541 


\section{Figure Captions}

Fig.1. Dependence of chemical potentials and temperature on time for $S-S$ collision initial condition. Solid, long-dashed and dashed lines mean the temperature, the strangeness chemical potential and the baryon chemical potential respectively.

Fig.2. Time - dependence of the square of the sound velocity for the expanding hadron gas. Long-dashed and solid lines mean the square of the sound velocity for the pion gas and for the baryonless hadron case respectively. 


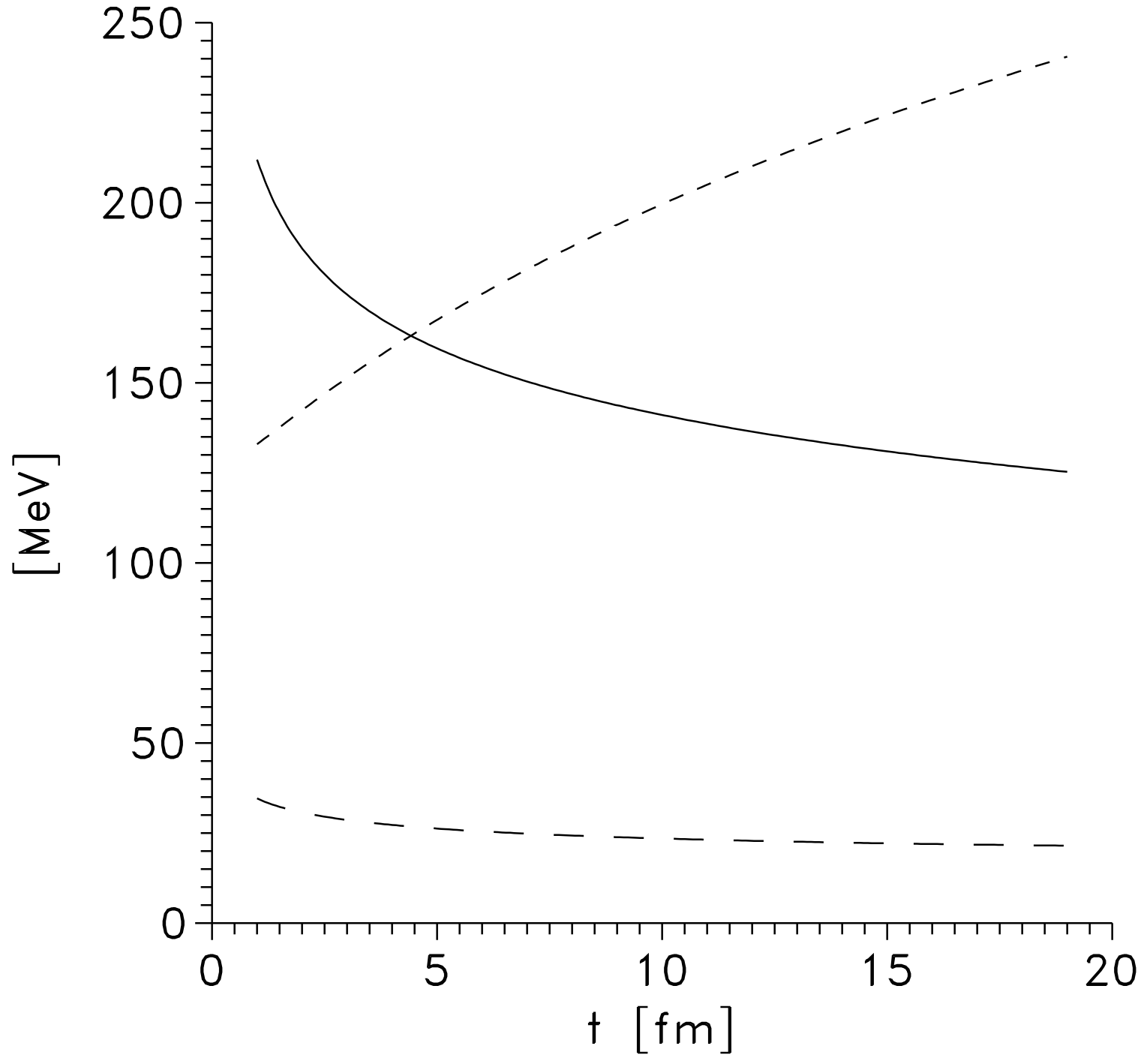




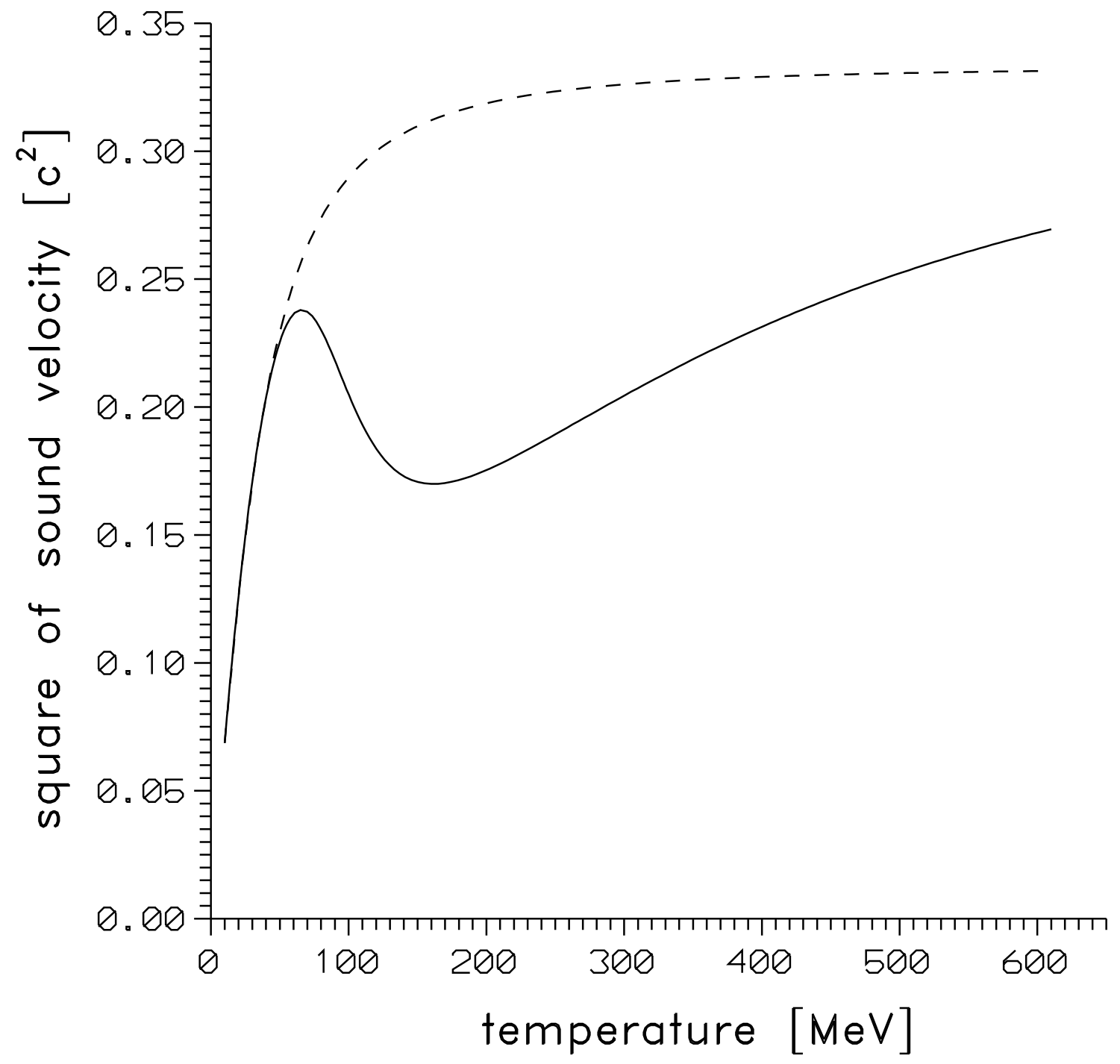


This figure "fig1-1.png" is available in "png" format from: http://arxiv.org/ps/hep-ph/9411346v1 
This figure "fig1-2.png" is available in "png" format from: http://arxiv.org/ps/hep-ph/9411346v1 